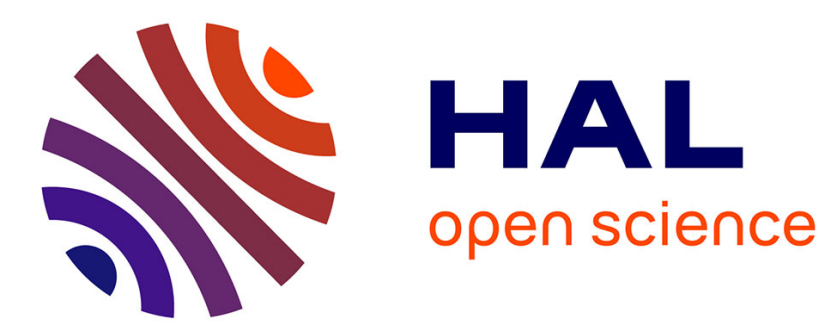

\title{
Pentecôtismes et esprit d'entreprise en Haïti
}

Nathalie Luca

\section{To cite this version:}

Nathalie Luca. Pentecôtismes et esprit d'entreprise en Haïti. Archives de Sciences Sociales des Religions, 2016, 175, pp.87 - 109. 10.4000/assr.27917 . halshs-01821590

\section{HAL Id: halshs-01821590 \\ https://shs.hal.science/halshs-01821590}

Submitted on 22 Jun 2018

HAL is a multi-disciplinary open access archive for the deposit and dissemination of scientific research documents, whether they are published or not. The documents may come from teaching and research institutions in France or abroad, or from public or private research centers.
L'archive ouverte pluridisciplinaire HAL, est destinée au dépôt et à la diffusion de documents scientifiques de niveau recherche, publiés ou non, émanant des établissements d'enseignement et de recherche français ou étrangers, des laboratoires publics ou privés. 


\section{Archives de sciences sociales des religions}

175 | juillet-septembre 2016

Figures de l'entrepreneur religieux - Vatican II : un concile pour le monde?

\section{Pentecôtismes et esprit d'entreprise en Haïti}

Pentecostalisms and entrepreneurial spirit in Haiti

Pentecostalismos y espíritu de empresa en Haití

Nathalie Luca

\section{revues.org}

Édition électronique

URL : http://assr.revues.org/27917

DOI : $10.4000 /$ assr. 27917

ISSN : $1777-5825$

\section{Éditeur \\ Éditions de l'EHESS}

Édition imprimée

Date de publication : 1 octobre 2016

Pagination : 87-109

ISBN : 978-2-7132-2518-5

ISSN : 0335-5985

Distribution électronique Cairn

CAIRN INFO

CHERCHER, REPÉRER, AVANCER.

Référence électronique

Nathalie Luca, «Pentecôtismes et esprit d'entreprise en Haïti », Archives de sciences sociales des religions [En ligne], 175 | juillet-septembre 2016, mis en ligne le 01 octobre 2018, consulté le 23 juin 2017. URL : http://assr.revues.org/27917 ; DOI : 10.4000/assr.27917 


\section{Nathalie Luca}

\section{Pentecôtismes et esprit d'entreprise en Haïti}

En 2007, je commençais une étude sur l'essor international d'entreprises de vente directe dont les réseaux de vendeurs s'appuyaient, pour se développer, sur des réseaux de fidèles d'Églises néo-pentecôtistes. Produits à vendre dans une main, Bible dans l'autre, ceux-ci menaient de pair une activité professionnelle et prosélyte. Je comparais leur réception dans deux contextes nationaux très différents : la France et la Corée du Sud, quand mon collègue L. Hurbon, anthropologue spécialiste d'Haït, m'apprit que l'une de ces entreprises, AMWAY (American Way) avait connu une envolée de courte durée à Port-au-Prince et m'invita à séjourner chez lui pour en comprendre l'échec. Je fis ainsi deux premiers séjours à Port-au-Prince, en 2007 et en 2009, durant lesquels je visitais plusieurs églises pentecôtistes et constatais leur animosité envers AMWAY : la réussite économique y était très clairement dévalorisée. J'y retournais en 2014, suite à l'invitation des professeurs L. A. Clorméus et J. P. Byron de la faculté d'ethnologie de l'Université d'État d'Haïti ${ }^{1}$. J'observais alors une sensible évolution des discours et des pratiques pentecôtistes. Certains pasteurs encourageaient les fidèles à s'engager dans le devenir social et économique de leur pays. Le séisme meurtrier de 2010 semblait modifier leur rapport au monde. Cet article repose donc sur des observations de terrain et une série d'entretiens menées en Haïti, avant et après le séisme, pré et post apocalypse, qui démontrent la remarquable plasticité des Églises néo-pentecôtistes, capables de changer de discours, de postures et d'attitudes vis-à-vis de l'Occident (et des États-Unis en particulier), mais aussi vis-à-vis du vaudou et de la relation à construire avec l'ensemble des Haïtiens.

Ce que j'ai aperçu de ce pays durant ces trois séjours est certes limité, mais la mise en perspective avec mes terrains antérieurs, et en particulier avec la Corée

1. Je remercie très chaleureusement mes trois collègues, ainsi que l'Université d'État d'Haïti d'avoir rendu cette étude possible. Grâce à eux, j'ai découvert un pays terriblement attachant où je n'imaginais pas aller et où je n'imagine pas ne plus retourner. Je remercie également les pasteurs qui m'ont accueillie dans leurs églises et l'ensemble des Haïtiens qui ont bien voulu se confier à moi. 
du Sud, cette grande puissance internationale sortie en un temps record du sousdéveloppement, m'apporte un éclairage particulier de la situation économicoreligieuse haïtienne. Il est complété par la comparaison qui s'impose avec d'autres études de cas de ce volume, et en particulier avec celle sur l'Éthiopie présentée par E. Fantini. Comme la Corée du Sud, l'Éthiopie est un pays à État fort, également capable d'impulser l'une des croissances économiques les plus importantes de la région. Dans les deux cas, cela a été profitable au pentecôtisme qui s'est développé en diffusant un message spirituel particulièrement favorable à l'esprit d'entreprise, même si cela ne se traduit pas par le même type de relations avec l'État. En Corée du Sud, les Églises ont soutenu la politique du gouvernement qui en échange, leur a laissé une place importante dans l'espace public. En Éthiopie, le gouvernement y est tout à fait hostile, tant il redoute leur politisation. Tout au contraire, Haïti se distingue par sa quasi-absence d'État et son incapacité à sortir d'une situation de pauvreté qui ne cesse d'empirer depuis plus d'un demisiècle et fait d'Haïti l'un des pays les plus pauvres du monde. Ainsi A. Corten écrivait déjà en 2001 :

Haïti ne correspond pas - au moins aujourd'hui - à une situation de domination totalitaire mais la désolation y produit des effets analogues. La désolation comme destruction de la vie privée et comme privation d'une expérience sensible communautaire est en fait un récit où il n'y a aucune quête d'un objet de valeur possible. Elle raconte une histoire où aucun "faire " n'est possible. Il y a une voix qui parle, mais c'est la voix de la fatalité (A. Corten : 2001, 41-42).

Cette double absence de richesse et d'État rejaillit sur le type de pentecôtisme qui se répand en Haïti, d'un côté, particulièrement attentiste et tourné vers la fin du monde, de l'autre, faisant fonction d'État au niveau local. Sans autorité surplombante, les Haïtiens peinent à trouver un moteur susceptible de les mobiliser et de les faire avancer. Il leur manque un désir, un rêve, un projet collectif. Bien que très peu utilisée en sciences sociales, la notion de désir est convoquée ici, de façon expérimentale, pour tenter d'éclairer les différents types de pentecôtismes observés sur le terrain avant et après le séisme de 2010 et d'approcher la façon dont le néolibéralisme pénètre en Haïti. La forme idéale de développement du néolibéralisme voudrait que l'État soit soumis à l'économie : qu'il y ait formation "d'un État sous surveillance de marché plutôt qu'un marché sous surveillance de l'État» (M. Foucault, 2004 : 120). Ainsi, lorsque l'on connaît le lien entre néo-pentecôtisme et néolibéralisme (également rappelé par E. Fantini) on peut se demander quelles sont les conséquences de la montée du néo-pentecôtisme en Haïti où l'État est déjà si faible. Comment résiste-t-il ? Comment, dans sa relative impuissance, gère-t-il le désir de reprendre son destin en main que ce courant religieux stimule chez les fidèles en même temps qu'il suscite un élan entrepreneurial autrement absent ? Ce faisant, le néo-pentecôtisme participe à la formation de l'homo-oeconomicus néolibéral, qui n'est autre, selon Foucault qu'un « entrepreneur de lui-même » dans le sens où il est « à lui-même son propre 
capital [et] pour lui-même son propre producteur. [...] Il produit tout simplement sa propre satisfaction »(M. Foucault, 2004 : 232).

Dans son ouvrage Capitalisme, désir et servitude. Marx et Spinoza (2010), F. Lordon reprend le concept de conatus de Spinoza pour expliquer comment l'adoption par leurs employés du "désir-maître » des chefs d'entreprise dans les sociétés occidentales ultralibérales réduit ces employés à un état de servitude dont ils demeurent inconscients tant qu'ils parviennent à croire que ce désir est le leur. Selon Spinoza, le conatus, c'est « la force d'exister »(F. Lordon, $2010: 17$ ), " l'élan » (32), "l'énergie fondamentale qui habite les corps et les met en mouvement » (17), une énergie tirée du besoin primordial de tout individu de désirer. Cependant le désir se construit. Il se forge à partir de la relation que l'individu, le groupe ou la société développe avec l'autre si bien que ce sentiment entretient, paradoxalement, un rapport d'altérité avec celui qui le porte. La mise en mouvement de l'individu, du groupe ou de la société dépend de la capacité de chacun de s'approprier un désir nécessairement né du rapport à un autre. C'est ce que R. Girard appelle "le désir mimétique ", en ce qu'il implique toujours un tiers. En Haïti, le tiers, c'est l'Occidental, et le rapport avec cet autre est à la fois de résistance, d'admiration méfiante et d'adaptation. La culture de résistance est le propre du vaudou, religion des pauvres par l'intermédiaire de laquelle " [ils] créent un espace autre, qui coexiste avec celui d'une expérience sans illusion [...] Ils déjouent la fatalité de l'ordre établi [...]. [Ils] contest[ent] aux hiérarchies du pouvoir et du savoir leur "raison” [...] [Ils] s'oppos[ent] à l'assimilation » (M. de Certeau, 1990 : 34-35). Les anthropologues qui s'intéressent au rôle du vaudou dans la société haïtienne pointent tous, à la manière de Karen E. Richman, sa fonction de résistance au système capitaliste de production (K. E. Richman, 2005). Cette résistance se retrouve dans les Églises pentecôtistes pré-millénaristes qui attendent la Fin dans l'espoir qu'elle leur apporte une revanche sur le monde capitaliste et matérialiste, en même temps qu'ils accusent les pratiquants du vaudou d'être les responsables diaboliques de la déchéance d'Haïti. L'admiration méfiante est du côté des élites catholiques qui veulent à la fois imiter l'Occidental, être reconnues par lui et s'en démarquer, ce qu'elles font notamment en gardant leur distance avec le néolibéralisme américain. Enfin, l'adaptation est la position des néo-pentecôtistes dont les églises se développent depuis le séisme de 2010 et dont les fidèles sont considérés comme des "traîtres à la nation ", tant par les pratiquants du vaudou que par les pentecôtistes, parce qu'ils leur apparaissent comme parfaitement alignés sur le "désir-maître " des Occidentaux. Désir de résistance, désir de reconnaissance, désir de réussite économique se construisent en tension, divisent les citoyens et rendent particulièrement difficile le tracé des frontières symboliques de l' " haïtianité ». Si cette expression est particulièrement revendiquée par les pratiquants du vaudou qui l'associent à l'esprit de résistance intrinsèque à leur culture, les néo-pentecôtistes, quant à eux, militent pour la naissance d'une « nouvelle Haïti » dans laquelle le vaudou cèderait sa place aux 
valeurs libérales américaines. Après le séisme, une position plus conciliante semble se dessiner et avec elle apparaît une figure originale, haïtienne, de pasteurentrepreneur.

\section{Un désir de liberté trop vite contraint}

Haïti fut la première République noire. L'île fut découverte par Christophe Colomb en 1492, sa partie occidentale cédée à la France en 1697. Sa population autochtone, rapidement décimée, fut remplacée par des esclaves noirs importés d'Afrique dès le XVI siècle et obligés de vivre sur cette terre étrangère qu'ils firent progressivement leur. C'est ainsi qu'à l'aube du XIX siècle, dans un mouvement de révolte impulsé par un impétueux désir de liberté dont les Français avaient mésestimé la puissance, les esclaves africains, les noirs affranchis et leurs descendants gagnent leur indépendance et forment le peuple haïtien. On est en 1804. La jeune République fut aussitôt encadrée par les " élites intellectuelle et économique ", élites urbaines issues "d'anciens affranchis et de chefs militaires ayant participé à la guerre d’Indépendance » (L. A. Clorméus, 2012 : 59). Elles prirent le pouvoir sur une population rurale aux attentes non unifiées, peu instruite, mais investie d'une "haïtianité » toute neuve, dont les contours, s'ils restaient à préciser, étaient déjà marqués par un esprit de résistance face à l'Occident, esprit qui prenait corps dans le vaudou. Les élites, quant à elles, furent séduites par les valeurs occidentales. Elles virent dans les institutions étatiques et religieuses de l'ancien colonisateur un véhicule capable de conduire leur pays sur la voie d'une indépendance durable et de lui apporter le respect des autres nations ${ }^{2}$. Haïti s'est ainsi construite en adoptant le modèle français, faisant du catholicisme la religion d'État sans se préoccuper de la place à donner au vaudou que toutes les classes sociales - celles au pouvoir y comprises - continuaient de pratiquer plus ou moins clandestinement selon les époques. Faute de temps et de maturité politique pour penser un modèle alternatif, le désir de liberté, intrinsèque au fondement du peuple haïtien, se mua en désir d'une minorité dominante d'être reconnue par l'Autre. L'élite, conduite par Toussaint Louverture (ancien esclave affranchi en 1777) imposa par le haut des normes et des valeurs qui niaient les besoins, les pratiques et les demandes du plus grand nombre, prenant le risque d'étouffer la pulsion par laquelle le peuple s'était éveillé à lui-même. Ainsi, constate L. Hurbon : "Si le vaudou a pu servir de ciment au lien social chez les esclaves en lutte contre les colons [...], il apparaît comme une source de division au regard de Toussaint, car des prêtres vaudou sont souvent à la tête de bandes

2. Notons, avec L. Hurbon, que le «clergé n'était pas tout entier dévoué à la cause esclavagiste ». On sait notamment que les jésuites ont été expulsés de Saint Domingue en 1763, pour encourager un esprit de révolte. Par ailleurs, explique l'auteur, bien des prêtres ont pris " au moment des troubles [...] le camp des esclaves insurgés »(2004 : 106). 
maronnes parallèles à l'autorité centrale. Toussaint choisit de les poursuivre et c'est le catholicisme qui peut selon lui assainir les mœurs, produire une certaine homogénéité sociale, donner le sens de la famille en favorisant les mariages, assurer l'éducation du peuple» (L. Hurbon, 2004 : 125). De fait, Haïti s'est développée sur un ersatz d'État, laissant la majorité de la population vivre dans des conditions proches de l'esclavage. Au début du $\mathrm{XX}^{\mathrm{e}}$ siècle, on ne comptait encore qu' " un seul prêtre haïtien dans le clergé » (idem : 194), tous les autres étant français, majoritairement bretons. Il fallut attendre les années 1960 pour que se forme un clergé haïtien et finalement, une véritable Église haïtienne.

Plusieurs jeunes intellectuels se demandent aujourd'hui si le protestantisme n'aurait pas pu offrir une alternative mieux adaptée à la population rurale, et par conséquent plus à même d'unifier la société haïtienne. L'État et l'Église catholique sembleraient cependant avoir tenté de bloquer la progression des missions protestantes. Ainsi, selon L. A. Clorméus (2012) les trois campagnes antisuperstitieuses, révélatrices des fortes tensions internes au pays, qui ont marqué la première moitié du XXe siècle $(1896-1900$; 1911-1912 et 1939-1942), avaient pour cible officielle les pratiquants du vaudou mais auraient été également dirigées contre les protestants et les francs-maçons dont l'Église catholique redoutait la concurrence. Les premiers missionnaires protestants arrivèrent sur l'île dès 1816 , mais la préférence de l'État pour le catholicisme leur valut une forte hostilité. On sait que les missions protestantes ont pu être utilisées en Afrique, au Groenland ou dans les Amériques pour assujettir les populations au pouvoir colonial. Cependant lorsque les Églises étaient dirigées par des locaux, elles ont aussi pu participer à renforcer leur désir d'indépendance ou bien encore, comme en Corée, accélérer la mise en place de réformes sociales d'envergure ${ }^{3}$. Contestant à la fois le catholicisme et le vaudou (tous deux considérés comme fétichistes), on peut en tout cas postuler qu'en Haïti, ces missions, ont proposé, avec d'autres - la franc-maçonnerie par exemple, qui luttait précisément pour un clergé national une troisième voie qui bousculait les habitudes du néophyte, l'obligeaient à effectuer une véritable conversion (non nécessaire dans le catholicisme) et à s'investir sérieusement dans la nouvelle croyance, ce qui comportait un danger assumé, celui d'appartenir à une minorité ostracisée. Dans ces conditions, la conversion pouvait représenter pour le fidèle l'occasion de faire un choix difficile peut-être apte à lui rendre la flamme et l'énergie nécessaires à le mettre en mouvement au nom de nouveaux idéaux qu'ils faisaient sien - même si ceux-ci étaient également

3. Le projet d'une Église nationale adossée au pouvoir politique a été formulé par Soulouque (l'un des dictateurs haïtiens les plus redoutés) dès 1849 . Un concordat est signé après la chute de Soulouque en 1859, avec le président Geffrard. "Désormais, une Église toute faite, c'est-à-dire, la chrétienté, peut s'installer dans le pays, avec le maximum de privilèges, mais adossée au pouvoir politique. Aux congrégations religieuses [...] seront confiées éducation et instruction. Elles devront mettre en œuvre et fournir au pays une élite formée à l'occidentale » (L. Hurbon, 2004 : 142). 
empruntés à l'Occident. Pour le dire autrement, on peut se demander si ce choix ne leur permettait pas de recouvrer leur capacité d'entreprendre mise en œuvre lors de la Révolution. Cette hypothèse peut être posée précisément parce que les missions protestantes nommaient des pasteurs haïtiens. Elles formaient des communautés autogérées dont il est possible de déduire qu'elles étaient responsabilisées quant à leur devenir, celui de leur famille, et pourquoi pas, celui de leur nation. Ce que le gouvernement et l'Église catholique imposaient par le haut et par le biais d'un clergé étranger, les Églises protestantes l'auraient proposé par le bas et par le biais de pasteurs locaux, créant ainsi les conditions nécessaires à la mobilisation des classes, les Églises baptistes, historiquement majoritaires en Haïti ${ }^{4}$, mais aussi le méthodisme, l'épiscopalisme et l'anglicanisme, qui sont les plus anciennes Églises protestantes d'Haïti étant parvenues à toucher «les couches aisées et moyennes de la population » (A. Corten, $2001: 80$ ).

Cela expliquerait pourquoi le protestantisme fut ressenti comme un obstacle à combattre pour le clergé catholique et l'État haïtien. Il était susceptible de remettre au peuple la prise en charge de ses croyances et actions et par conséquent la question de son devenir social et national; de replacer l'individu dans son statut d'acteur citoyen là où les dictatures haïtiennes, soutenues par l'Église catholique, tentèrent par la force ou la menace de mettre sous cloche son désir de liberté. L. A. Clorméus rappelle que le wesleyen Louis-Joseph Janvier écrivait déjà en 1886 :

Par pur patriotisme, non par prosélytisme d'aucune sorte, chacun doit se répéter que la religion protestante peut devenir un puissant facteur de développement social en Haïti parce qu'elle est supérieure au point de vue des résultats économiques et peut être nationale [...] Le protestantisme est plus national, moins dangereux qu'un catholicisme sans clergé national ; celui-ci ne peut avoir qu'une existence anormale, factice ; il ne saurait durer à moins que le pays ne veuille perdre son indépendance politique ${ }^{5}$.

Que le protestantisme ait été ou non une meilleure solution, il apparaît en tout cas qu'en imposant le catholicisme comme religion d'État, l'élite haitienne a nui à la valorisation du peuple et à la prise de conscience de son caractère exceptionnel, deux éléments dont Benedict Anderson a montré l'importance dans la construction des frontières symboliques nationales (Anderson, 1996). Elle a introduit le sentiment d'une infériorité ethnoculturelle et empêché la formation d'un équilibre social, économique et politique. Cette dévalorisation a privé le pays de repères unificateurs. Un siècle après son indépendance, l'instabilité du pays (que la France a largement entretenue) conduit à l'occupation américaine (1915-1934).

4. Le protestantisme est arrivé en Haïti « dans le cadre du mouvement pour l'abolition de l'esclavage dans les colonies (abolition proclamée en 1838) [...] Des pasteurs baptistes anglais et jamaïquains ainsi que des pasteurs noirs américains débarquent en Haïti " (A. Corten, 2001 : 77).

5. Louis-Joseph Janvier, Les Constitutions d'Haïti (1801-1885), C. Marpon et E. Flammarion Libraires Éditeurs, Paris, 1886, p. 286 et 614, cité par L. A. Clorméus (2012: 174). 


\section{Une situation peu propice à entreprendre : attendre la fin du monde}

La présence des Américains a ouvert la route aux missions protestantes. C'est durant cette période, en 1928, que le pentecôtisme est arrivé sur l'île. A. Corten souligne néanmoins qu'il s'y est développé "sans interférence notable avec [l'occupation] » (2014:119). Il faut constater en effet que la spécificité du pentecôtisme est d'avoir su, dès le départ, recruter "des gens des classes défavorisées [en] se distingu[ant] par ses utopies égalitaristes » (L. A. Clorméus, 2012 : 331). Il allait dans le sens d'une résistance au libéralisme et donc à l'esprit du capitalisme américain. En cela, il visait le même public que le vaudou et s'il en diabolisait les pratiques, c'est pour convertir dans ses rangs et non pour s'aligner sur les valeurs occidentales. Ainsi, les églises pentecôtistes haïtiennes que j'ai visitées à l'occasion de mes deux premiers séjours mettaient l'accent sur une théologie extra-mondaine pré-millénariste ${ }^{6}$. Les croyants plaçaient leur espoir dans la survenue prochaine de la fin du monde. Déshérités et en grande souffrance dans ce monde-ci, ils espéraient être les élus d'un temps post-apocalyptique. Aucune vision prometteuse de l'avenir dans laquelle s'investir ne leur était proposée. Comme le constate également L. A. Clorméus, "La pauvreté passait pour une épreuve terrestre contre laquelle [lutter] était moins profitable que se consacrer à Dieu qui promet le paradis céleste à ses fidèles " (2012: 331). Les pasteurs expliquaient les malheurs écologiques, sociaux et politiques comme autant de bienfaits de Dieu et rappelaient qu'avant d'être sanctifié, Jésus avait été brisé :

Il n'y a pas de plénitude sans brisement [...] Après l'humiliation vient la sanctification [...] Vive la sècheresse du monde, car elle nous permet d'ouvrir nos cœurs et de boire Sa Parole!

La souffrance étant le signe de la fin des temps, il fallait attendre dans la résignation :

Mieux vaut souffrir encore que de perdre mon âme.

Ces attitudes croyantes étaient en tout point opposées à celles des Églises ostensiblement proaméricaines de Corée du Sud qui encourageaient les fidèles à être les acteurs du développement économique de leur pays, seul à même de prouver l'action bienfaitrice de Dieu sur leur nation. C'est bien là ce qui sépare pentecôtisme et néo-pentecôtisme, même si une gradation et des passerelles existent entre les deux courants : dans une perspective idéal-typique, on peut dire que le premier, pré-millénariste, vise un retrait du monde considéré comme diabolique, quand le second, post-millénariste, considère l'action et la réussite dans le monde comme preuve de la présence et de l'amour divin; le premier anéantit désir et vision d'avenir, le second cultive rêves et projections dans un

6. Ce constat n'est pas généralisable. Certaines Églises pentecôtistes s'adressent à un public socialement mieux inséré et s'adaptent à des demandes plus pragmatiques et dirigées vers ce monde-ci. 
futur meilleur qui se construit grâce à l'investissement des fidèles dans des activités économique, sociale et politique :

Le Royaume étant déjà de ce monde, il est donc nécessaire de poursuivre sa conquête afin qu'elle soit achevée lors de la seconde venue du Christ. C'est pourquoi, alors que le pentecôtisme prône un retrait du monde ce qui le conduit à ne guère s'intéresser aux réalités sociales qui lui sont extérieures, et à se montrer très réticent à l'égard de l'engagement politique, le néo-pentecôtisme campe quant à lui sur un projet clairement énoncé de transformation du monde (J. Garcia-Ruiz, P. Michel, 2012 : 92).

C'est précisément par des réseaux néo-pentecôtistes qu'a pénétré, en Haïti dans les années 1990, AMWAY (American Way), une entreprise de vente directe par réseaux apparue aux États-Unis dans les années 1950. Son fonctionnement repose sur la formation de distributeurs indépendants qui développent leur affaire à partir de la fidélisation d'une clientèle et la construction d'un réseau de vendeurs qui s'étend au niveau local, national ou international. Il n'y a aucun salariat, aucune rémunération fixe. Chacun gagne en fonction de ses ventes et de l'ampleur de son réseau. Il lui faut donc acquérir un esprit d'entreprise, c'est-à-dire, un certain goût du risque et une aptitude à convaincre. En Haïti, AMWAY proposait des produits de consommation courante (casseroles, savons, dentifrices, crèmes de soin, etc.), mais aussi de l'électroménager (télévisions, magnétoscopes, etc.), s'adaptant à la demande de classes sociales hétérogènes. Selon mes informateurs, elle aurait réuni plus d'un millier de distributeurs avant de s'effondrer dans les années 2000. En 2009, ils n'étaient pas plus d'une cinquantaine. L'entreprise est arrivée sur l'île par le biais de la diaspora haïtienne dont une partie fréquente les églises néo-pentecôtistes et s'engage dans ce type d'activité rémunératrice. Pendant quelques années, les distributeurs de Port-au-Prince ont insufflé un désir de réussite et une vision d'avenir à leurs équipes recrutées au sein même des communautés pentecôtistes en valorisant un complet changement d'attitude visà-vis du sens théologique à donner à la pauvreté et à la possibilité d'en sortir. Lors des réunions, dont certaines avaient lieu le dimanche aux mêmes heures que le culte, au grand dam des pasteurs, les distributeurs priaient et remerciaient Dieu avec ferveur avant de parler produits ou construction de réseaux.

Un ancien distributeur : "Avec ma femme, nous assistions religieusement aux réunions. On croyait tellement aux leaders qui avaient déjà réussi. On nous montrait des chèques et on rêvait de ces sommes, de l'idée de créer une entreprise multinationale en partant de zéro. Au moment où les problèmes ont commencé, on avait un réseau de plus de quarante personnes. Notre meilleur chèque était de 850 dollars US. On pouvait développer un leadership. C'est tout ça qui faisait la magie. Il y a aussi les livres qu'on lisait. Ça, c'était très, très important. Et puis oui, on priait. On priait, mais de façon universelle. Il y avait des croyances très variées. Parfois, on récitait quelques versets. On pouvait toujours demander à quelqu'un de prier. Il arrivait qu'un maître de cérémonie démarre la réunion sans prier, mais quelqu'un lui disait alors qu'on n'était pas encore prêt. Parfois, c'était une simple prière. Parfois, nous répétions ensemble un psaume. On remerciait le Tout-Puissant de nous avoir permis de rencontrer une entreprise si extraordinaire. On requérait son assistance; de nous aider à 
parler pour nous; d'ouvrir nos esprits, notre intelligence. Avant de partir, on priait alors pour demander sa protection et pour qu'il nous aide à réaliser nos activités jusqu'à la prochaine réunion».

AMWAY s'est effondrée pour cause de scissions internes et pour des raisons externes, dont la plus importante fut la réforme soudaine et à effet immédiat des taxes douanières. Les distributeurs, qui importaient leurs marchandises des États-Unis, ont vu leurs commandes bloquées en douane. La date de péremption de certains produits de consommation a expiré ; il a fallu rembourser les clients non livrés et les distributeurs ont perdu leur argent. Cela a rompu le climat de confiance indispensable au fonctionnement de l'activité et, même si la situation s'est stabilisée ensuite, les réseaux se sont défaits et les taxes douanières sont devenues trop élevées pour que l'affaire demeure rentable. La réforme de l'État avait cassé la dynamique. Cette situation n'est pas propre à la vente directe par réseaux. Elle est généralement critiquée par les entrepreneurs haïtiens :

Marc-Antoine Acra, président d'Acra Industries: "Nous avons besoin de stabilité politique. Sinon, personne n'investira. L'État haïtien se contente de nous taxer. Il n’offre pas le cadre pour développer les sociétés » (P. Woods et A. Robert, 2013 : XX).

D’autres marketings de réseaux ont bien tenté de se développer localement et certains jeunes haïtiens ayant fait leurs études en Occident, peu concernés par les discours religieux, ont voulu créer dans un cadre très professionnel des entreprises de distribution à partir de produits locaux, comme le rhum. Ils se sont heurtés au refus, de la part des producteurs qu'ils démarchaient, de prendre le risque d'augmenter brutalement leur production dans une telle situation d'instabilité législative. Mais le désir et l'espoir restaient en suspens, et ces réseaux continuèrent de susciter bien des critiques dans les milieux pentecôtistes, y compris, parfois, directement durant le culte. Un pasteur m'expliquait :

Certains voient, dans ces réseaux, la manifestation de Satan qui veut vous séduire, vous conduire vers l'erreur, vers un système qui fonctionne pour lui-même, pas pour vous. C'est le mal de la Bête. Tout repose sur l'illusion, la naïveté, les fausses promesses. Ils essaient de capter les esprits débiles, car les personnes clairvoyantes ne vont pas facilement entrer dans ce mouvement. Ils disent former une communauté soudée, mais ce n'est que superficiel; c'est professionnel et l'amitié tombe dès que l'affaire s'arrête. Beaucoup de personnes étaient très optimistes au départ, mais avec le temps elles ont été déçues et sont devenues hostiles. Elles sont revenues chez nous.

AMWAY a été ressentie comme un risque sérieux de concurrence. Les pasteurs étaient directement démarchés et certains entraînaient dans l'affaire toute leur assemblée. Les nombreuses dénominations pentecôtistes ne comprenaient pas pourquoi l'entreprise tenait ses réunions le dimanche, obligeant les fidèles à choisir entre le culte ou ses meetings. D'où la montée d'une contestation qui alla jusqu'à interdire aux distributeurs de participer à la vie paroissiale :

Un pasteur pentecôtiste : «On est né dans la pauvreté, on grandit dans la pauvreté et on va mourir dans la pauvreté ». 
Certes, AMWAY représentait le rêve américain. Surfant sur les réseaux néopentecôtistes pour se développer à l'international, ce type d'entreprise proposait un moyen efficace "de produire un "individu compatible" avec les règles en vigueur dans l'univers marchand, qui s'appliquent là, complètement, en ce qu'elles dessinent et redessinent en permanence le profil d'un individu producteur/ consommateur aussi adapté que possible aux logiques du marché » (Garcia-Ruiz, Michel, 2012 : 36). Mais cet "individu compatible» n'était pas imposé aux Haïtiens de l'extérieur, par le haut, il était proposé à chacun comme une possibilité de réaliser son propre rêve, d'imaginer sa société de demain, bref, de s'investir, d'entreprendre, de se mettre en mouvement pour un désir qu'il pouvait s'approprier et qui le transformait. On retrouve là le cœur de l'analyse de Lordon : celui-ci se demande en effet «comment certains salariés [nous dirons ici les laissés-pour-compte du développement économique] en viennent à faire cause commune avec le capitalisme, pourquoi [ils] marchent avec lui » (Lordon, 2010 : 54). La force du néo-pentecôtisme est très précisément de trouver des arguments permettant à chaque fidèle de se laisser capturer par le " désir-maitre " des néolibéraux américains, de se convaincre qu'il est le sien et qu'il doit se donner les moyens de le réaliser, c'est-à-dire, de s'enrichir, l'enrichissement devenant le maître-mot de la réussite. Cette appropriation du désir était facilitée par un système de vente comme AMWAY non seulement parce qu'il n'y avait pas de patron, mais parce qu'il n'était pas nécessaire, pour se lancer, d'un apport initial. Les distributeurs, dans leur grande majorité, bénéficiaient d'une égalité de fait : ils n'avaient que leur énergie à mettre à la disposition de leur réussite. Les prières en début ou fin de réunion, les discours des animateurs d'équipe avaient pour unique fonction de déposer dans l'esprit du distributeur un " affect gai ", celui de l'espoir, capable de prendre la place de l' " affect triste » diffusé par les pasteurs pentecôtistes qui distillaient la crainte du jugement dernier et recommandaient de se soumettre à son état de pauvreté. Ces prières permettaient la «mobilisation, au sens le plus littéral de savoir ce qui fait mouvoir les corps, c'est-à-dire ce qui induit les énergies des conatus à faire ceci ou cela et avec quelle intensité. » (F. Lordon, 2010 : 48). Pour Spinoza, ces énergies sont les « affects ». Ceux-ci dirigent les désirs dans un sens où un autre selon qu'ils sont positifs ou négatifs.

Une fleuriste : "Grâce à ce nouveau job, les jeunes apprenaient à parler, à s'exprimer, à s'habiller, ce que la société ne leur offre pas. Ça les obligeait à acquérir certaines valeurs sans lesquelles ce système ne marche pas. Peut-être que beaucoup n'ont pas gagné grand-chose sur le plan financier, mais ils se battaient pour quelque chose puisqu'ils n'ont rien! Ils se levaient le matin avec une lueur que rien d'autre ne leur avait donnée. C'est pas rien, ça!»

On peut se demander pourquoi le gouvernement en place a arrêté cet élan entrepreneurial avec des lois régulant et limitant la circulation des biens. Les distributeurs pensaient qu'il s'était sciemment opposé au développement d'une entreprise façonnée par l'esprit néolibéral américain si défavorable à toute forme 
de régulation étatique. Il est possible de voir là, en effet, une modalité d'existence et de résistance de l'État haïtien face au néolibéralisme américain alors même que son impuissance politique le soumet déjà aux ONG, que la sécurité est très largement prise en charge par l'ONU à travers la MINUSTAH $^{7}$ (au grand dam de la population), et qu'il est incapable d'assurer la prospérité de la nation.

Que ce soit sur le plan de la santé, de l'éducation, de l'environnement, ou sur celui des associations féminines, ou encore de l'organisation des jeunes, les ONG donnent l'impression d'être des substituts de l'État. Comme si les rapports Église-État se déplaçaient et se transformaient en rapport État-ONG, sans pour autant que s'opère un changement véritable de la nature de l'État. Désormais, dans ce vide, tout peut prendre place. Les repères symboliques venant à défaillir, la société tout entière s'enfonce peu à peu dans l'anomie (L. Hurbon, 2004 : 259).

Le refus de la diffusion de l'idéologie capitaliste semble être une des valeurs les mieux partagées par les Haïtiens, par-delà les clivages entre pratiquants du vaudou, pentecôtistes et catholiques. C'est d'ailleurs là le regret de bien des chefs d'entreprise haïtiens :

Jerry Tardieu ${ }^{8}$, actionnaire de l'hôtel Oasis : "Il y a trente ans, nous avions une usine qui exportait le jus de mangue. Tout s'est effondré. Nous vivons dans un pays où les gens de la bourgeoisie sont perçus comme des rapaces. Il faut, pour reconstruire Haïti, que la mentalité change de part et d'autre»(P. Woods et A. Robert, $2013: \mathrm{XX}$ ).

Pour F. Lordon, cette résistance représenterait sans doute ici le souci des dominés ( les enrôlés » dans son vocabulaire), dont l'État haïtien, de se décaler du « désir-maître ", en l'occurrence ici, le désir de propagation du néolibéralisme de la plus grande puissance du monde, et certainement la plus envahissante en Haïti. Le néopentecôtisme associé à une entreprise américaine comme AMWAY qui fonctionnait de concert avec la théologie de la prospérité représentaient ensemble un seul et même mouvement œuvrant à faciliter " la colinéarité ", c'està-dire l'alignement du " désir des enrôlés sur le désir-maître ", ou bien encore, la capacité des dominés à désirer la même chose que les dominants. En bougeant les règles, en les corrigeant en fonction des effets qu'elles produisaient, l'État haïtien fit exactement le contraire de ce qui est attendu dans une économie néolibérale où la loi « doit définir un cadre à l'intérieur duquel chacun des agents économiques pourra décider en toute liberté, dans la mesure où, justement, chaque agent saura que le cadre légal qui est fixé à son action ne bougera pas » (M. Foucault, 2004 : 170). L'État a donc fait la seule chose par laquelle il pouvait encore efficacement résister à l'Occident.

7. Mission des Nations Unies pour la stabilisation d'Haïti, inaugurée en 2004 après le départ précipité du président Jean-Bertrand Aristide et composée en 2016 de 2366 militaires et 2374 policiers de l'ONU (pour une population globale en Haïti de 10 millions d'habitants).

8. J. Tardieu représente la commune de Pétion-Ville à la chambre des Députés. 


\section{L'élan d'après le séisme}

La présence des ONG s'est encore intensifiée suite au séisme du 12 janvier 2010, si meurtrier et destructeur qu'il semblait concrétiser la fin du monde annoncée, sauf que seule Haïti était touchée.

Sept sur l'échelle de Richter : un séisme équivalent à plusieurs bombes atomiques qui auraient explosé sous la terre. Le 12 janvier 2010, une maison sur trois a résisté. Si l'une a miraculeusement tenu, sans une vitre soufflée, l'autre s'est trouvée réduite à néant : un gigantesque jeu de hasard et de massacre qui a fait 230000 morts, 300000 blessés et 1,3 million de sans-abris. Sans compter les dégâts matériels : 7,8 milliards de dollars, $120 \%$ du PIB de l'année $2009^{9}$.

E. McAlister constate que la majorité des Églises évangéliques virent dans cette catastrophe « le début de l'apocalypse [...] Plusieurs Églises [organisèrent] d'importantes “croisades" [...] Une minorité d'extrémistes [affirmèrent] avoir reçu la révélation que Dieu était en colère contre les Haïtiens pour la corruption de leur gouvernement, les débauches sexuelles, la pratique du vaudou considérée comme forme d'idolâtrie. Ce [fut] d'ailleurs la position que le télévangéliste américain Pat Robertson [adopta] et qui [suscita] une grande controverse " (L. A. Clorméus, 2014 : 136).

Je revins en 2014, et bien que je ne sois pas restée longtemps, une chose me frappa : alors que j'imaginais ressentir l'apocalypse annoncée, c'est une lueur d'espoir qui m'apparut. Il ne faut certainement pas la généraliser à l'ensemble d'une population qui survit dans des conditions déplorables, mais son existence mérite qu'on s'y intéresse. Je rencontrai de jeunes intellectuels liés à de jeunes pasteurs, les uns et les autres ayant fait leurs études et sachant leur avenir assuré à l'étranger. Ils avaient fait le choix de rentrer pour participer à la reconstruction d'Haïti. Ils remettaient en question l'utilité même de l'État et critiquaient des ONG trop intrusives. Surtout, ils proposaient un discours et des actions de responsabilisation, s'opposant à la victimisation de leur peuple. Ils croyaient en l'avènement de "la nouvelle Haïti ", une nation qui émanerait non pas d'une attente passive vis-à-vis d'un État providence ou d'ONG bienfaitrices, mais d'initiatives prises au niveau local, partant de citoyens qui se regroupaient en petites structures. Celles que j'observai étaient pentecôtistes, mais ce n'étaient pas les seules. Une plus longue enquête de terrain serait nécessaire pour mieux appréhender l'ampleur de ces initiatives et la diversité de leurs auteurs. P. Woods et A. Robert, photographe et journaliste ayant réalisé en 2013 un très bel ouvrage de photos accompagnées de textes en créole et en français portant le titre Leta (L'État), manifestent en tout cas dans leur introduction le même étonnement :

Depuis trois ans, nous avons sillonné Haïti. Comme des milliers d'autres journalistes, comme des milliers d'autres photographes, nous inscrivons nos pas dans l'histoire des

9. Dans Le Courrier du mercredi 8 mai 2013 est reproduit un article du Monde diplomatique de mai 2013, intitulé « Haït dépecé par ses bienfaiteurs », par Céline Raffali. 
représentations d'une île toujours caractérisée par sa misère obscène, ses dictatures ubuesques, ses plans de sauvetage par l'international. Il ne s'agissait pas pour nous de transformer artificiellement un pays où plus des deux tiers de la population souffrent régulièrement de la faim en un espace marqué seulement par la créativité, la résistance, les parades imaginaires et l'humour permanent comme outil culturel de survie. Mais ce qui nous a frappés, plutôt que le désastre dont on nous avait abondamment prévenus, ce sont les ordres sous-jacents, les tactiques mises en œuvre par une population que l'on réduit en général à son statut de victime, l'incroyable cohérence d'une société dont les visiteurs s'obstinent à ne percevoir que le désordre (P. Woods et A. Robert, $2013: \mathrm{X})$.

Parmi les anthropologues, le travail de terrain d'Elisabeth McAlister conduit aussi à observer ces nouvelles stratégies de résistance au sein d'une Église pentecôtiste issue du Spiritual Mapping Movement, composée de 500 membres installés dans un camp de sinistrés et dirigée par la pasteure Yvette qui refuse l'aide humanitaire aussi souvent que possible pour développer une politique d'autosuffisance. La pauvreté et les malheurs - séisme compris - de ce pays y sont analysés en termes de punition, de réponse divine au pacte que les pratiquants du vaudou ont passé avec Satan. Il ne s'agit pas de s'identifier à des victimes, mais d'accepter le jugement divin et de faire en sorte que les actions des fidèles aillent désormais dans le sens attendu par Dieu. Celui-ci les a épargnés, non pas pour qu'ils se plaignent, mais pour qu'ils agissent et transforment leur pays en une Terre digne du Christ. Cette croyance les conduit à mettre en place une structure très efficace d'entre-aide mutuelle qui leur a permis de sortir du camp, de retrouver une maison bien plus rapidement que les autres sinistrés, de construire une église sans aucune aide extérieure, surtout, de reprendre en main leur destinée. Comme pour les églises pentecôtistes observées avant le séisme, l'argent reste un symbole largement satanisé des puissances occidentales, mais les fidèles ne sont plus dans une attente passive de la fin du monde. Ils se situent bien plutôt dans une espérance post-millénariste qui les conduit à adopter un mode d'action qui les valorise, sans pour autant que ni la pasteure Yvette ni les fidèles ne se comportent en entrepreneur. Simplement, le chef d'entreprise qui les fait bouger n'est plus l'étranger, l'ONG; ils l'ont choisi, et le reconnaissent en la personne de Jésus.

Money, the love of which is thought to be the root of all evil, is kept discretely out of the center of attention, and material aid is not give or received as such (E. McAlister, $2013: 28)$.

Mes observations menées auprès de deux Églises de type néo-pentecôtiste allèrent dans le même sens. La première paroisse se trouvait à Port-au-Prince, assez éloignée du centre-ville. Elle était au milieu de maisons à moitié construites, avec ou sans toit, et généralement sans vitre, sans portes, mais dans un quartier relativement calme. L'église elle-même n'avait pas de vitre, mais des barreaux aux fenêtres. Les murs étaient en ciment, rien n'était terminé, mais il y régnait un air de fête. Les fidèles avaient installé des rideaux et des fleurs en plastique qu'ils retireraient à la fin du culte. Ils étaient tous endimanchés. Des plus jeunes 
aux plus âgés, personne ne portait de mise négligée. Les femmes et les fillettes avaient les cheveux dégagés et portaient de jolies chaussures à talon. Elles étaient très élégantes. Je me serais cru dans une ville avec des trottoirs bien entretenus. Comment faisaient-elles pour marcher sur ces routes en si mauvais état ? Personne ne donnait l'impression de vivre un cauchemar quotidien. Si le lieu était des plus rudimentaires, la technologie de pointe était très présente : micros, instruments électroniques, deux belles enceintes, un rétroprojecteur affichant un powerpoint permettant de suivre le culte. Tout était fait pour oublier la pauvreté, le malheur. Les émotions étaient dignement retenues. Les fidèles n'étaient visiblement pas là pour pleurer, mais pour s'instruire et construire la société de demain :

Le pasteur Samuel Fligne: "Sur le plan économique, la participation de l'Église est de motiver des fonds, mais aussi d'enseigner aux fidèles les pratiques économiques : comment économiser ? Comment gérer les fonds? Comment et quand les utiliser ? C'est ainsi que nous pourrons produire des leaders bien instruits, bien formés, bien encadrés pour aider le pays à sortir de ce bourbier économique [...] Après avoir éduqué, sensibilisé l'homme haïtien, on est certain que l'Haïti de demain sera une Haïti possible. Et nous croyons tous dans la nouvelle Haïti. C'est pourquoi nous voulons une nouvelle Église pour une nouvelle Haïti, une nouvelle école pour une nouvelle Haïti, de nouveaux leaders pour une nouvelle Haïti. Notre génération peut ne pas en bénéficier, mais ce qui est certain, la nouvelle Haïti verra le jour quand même [...] Les jeunes de l'église peuvent contribuer à une Haïti prospère, juste, harmonieuse et équitable ».

Dans le sermon, il était question d'investissement, mais il s'agissait d'investir ses propres compétences; de générosité, mais pour mettre ses compétences au service de l'Église et de son prochain.

Ce n'est pas de la charité. Vous pouvez donner de la sagesse, du temps, du talent. Ce n'est pas qu'une question d'argent. Ce qui est important, c'est la manière de donner. Il faut investir avec proportion. Il faut soutenir les faibles. Vous êtes les gérants d'une œuvre dont Dieu est le propriétaire.

Le pasteur Fligne se considérait comme le gérant de son église et des talents de ses fidèles et se sentait pleinement responsable de son devenir. Il était actif, il avait une vision, un désir d'entreprendre, de faire bouger les lignes. Il n'y avait plus la résignation et l'attente passive d'avant le séisme. Il n'y avait pas non plus d'alignement sur un "désir-maître ". L'individu était certes responsabilisé et autorisé à rêver, à croire, à se projeter dans les habits d'un grand homme. Il demeurait quelque chose de l'état d'esprit insufflé par AMWAY mais avec une nuance importante : le gain d'argent n'était pas la finalité, ce qui permettait de garder ses distances-résistances avec les puissances dominantes. L'argent n'était même pas mentionné. Le rêve était à la fois plus ambitieux et plus noble ; plus politique et moins économique : il s'agissait davantage de se donner un rôle dans la construction d'une société civile que dans celle d'une entreprise :

Le pasteur Fligne : "L'avenir ne se construit pas lui-même, c'est à l'individu de construire son avenir, ce que moi-même je commence à faire. Je construis l'avenir dans 
l'enseignement, dans la formation de la pensée des jeunes que j'ai l'occasion de fréquenter, soit les jeunes ados, soit les pré-adultes [...] C'est ainsi que moi-même je forme l'avenir et je dessine mon avenir ».

La réussite économique n'était pas pour autant diabolisée. Le bien-être matériel était jugé utile et conforme à l'époque. Il ne s'agissait ni de célébrer une théologie de la prospérité, ni de défendre une théologie de la pauvreté. L'engagement se situait à un autre niveau. L'accent était mis sur la progression du capital humain et du capital social par le biais du travail afin d'apporter les fondements nécessaires à la croissance économique. En cela, la position du pasteur Fligne, comme celle des Églises dans lesquelles elle s'inscrivait, n'était pas antiaméricaine ou antioccidentale, elle ne se positionnait pas à rebours du néolibéralisme, elle préparait au contraire ses adeptes à y jouer un rôle, à en être, en se constituant déjà entrepreneurs d'eux-mêmes.

L'Église peut aider cette communauté à avoir une mentalité, à se libérer de la mentalité pauvre. À aborder le chemin de la richesse. Quoique la Bible ne préconise pas la prospérité, mais elle nous donne des moyens à suivre pour être prospère, notamment le travail. Si on veut être prospère, il faut bien travailler. Si on veut avoir des richesses, on doit travailler! Donc on ne doit pas venir ici en espérant des moyens financiers. À coup sûr vous n'aurez pas ici des moyens financiers. Mais si vous travaillez, vous aurez des moyens financiers.

\section{Et un jeune étudiant de confirmer :}

Un Haïtien, c'est un combattant. Peu importe les difficultés de la vie, peu importe les difficultés économiques, peu importe les difficultés sociales, les Haïtiens avancent [...] Je pense qu'un Haïtien doit penser avec un objectif dans la vie, et je pense que peu importent les moyens qu'ils ont, ils y arriveront. Et j'ai une pensée pour les jeunes. Peu importe les difficultés de la vie, je pense qu'ils arriveront. Un jour, ils arriveront [...] Des fois, ce qui arrive aux gens c'est qu'ils misent sur les conditions économiques qui sont précaires. Ils misent sur les conditions sociales. Mais des fois dans la vie, il faut être optimiste. Vous devez penser que vous y arriverez, peu importe les conditions. Car la vie, ce n'est pas avoir beaucoup d'argent, beaucoup de biens matériels, mais c'est que vous avez un objectif dans la vie et vous y arrivez.

La seconde église pentecôtiste visitée se trouvait dans une commune de 60000 habitants du département de l'Ouest d'Haïti, Ganthier, située à une vingtaine de kilomètres de Port-au-Prince, non loin de la frontière dominicaine. Cette église, au milieu de nulle part, en pleine campagne, difficile d'accès, n'avait pour l'heure que les fondations. Tout était à construire et c'est peut-être ici que nous trouvons le profil le plus évident d'un pasteur-entrepreneur. Issu d'une famille très modeste, le pasteur Valentin obtint un baccalauréat de philosophie, un diplôme de théologie et un autre de linguistique. Il finança ses études en donnant des cours d'anglais et d'espagnol dans le secondaire et fonda finalement sa propre école de langues en 2004, tout en devenant assistant-pasteur dans une grande église de Port-au-Prince qui réunissait entre 1500 à 2000 membres. Le séisme bouscula considérablement ses projets. Sa maison s'effondra et sa fille eut les 
deux jambes cassées. Il la conduisit à Saint-Domingue pour la soigner. Durant ce séjour, il s'engagea dans une ONG locale, la Fondation pour la paix :

En 2011, en juillet, on a eu l'épidémie de choléra qui est même devenue pandémique. J'ai travaillé pour la Fondation pour la paix qui est intervenue. J'ai dû visiter vingtdeux communautés reculées dans lesquelles le choléra battait son plein. Nous distribuions des gélules d'aquapure. Pour préparer l'aquapure, il faut attendre 30 minutes. Donc dans les communautés où j'ai fait la distribution, je prends toujours 30 minutes pour adorer [prier] avec elle. Quelle que soit la communauté, qu'elle soit chrétienne, qu'elle soit vaudouisante, ou quoi que ce soit. Je prends ces 30 minutes pour adorer parce que je ne voulais pas seulement passer, je voulais leur montrer que je crois en ce que je fais et donc, je suis la première personne à boire cette eau. Il y a eu cette communauté qui s'appelle Desroches. Je me souviens exactement, c'était la $19^{\mathrm{e}} \mathrm{com}-$ munauté que j'ai visitée. Pour une raison ou pour une autre, après 30 minutes, l'eau était prête, on a commencé à adorer avec la communauté et on ne pouvait plus s'arrêter. 30 minutes se sont écoulées. 40 minutes. Une heure. Toute la nuit on a continué à adorer, on sentait que le Saint-Esprit descendait sur nous. On oubliait totalement qu'on était là pour des raisons humanitaires. Et puis à la fin, il y a une dame qui m'a dit : " Pasteur, il n'y a aucune église dans notre communauté. Pouvez-vous nous rendre le service de venir ici une fois par mois pour prier avec nous. J'étais toujours lié à l'Église de Port-au-Prince, donc je ne pensais pas que ça allait être possible ».

En rentrant, il en parla avec sa femme, qui le soutint, puis avec le pasteur responsable de l'Église de Port-au-Prince. Celui-ci le découragea :

Il m'a dit: "Si vous faites une église ici, il n'y aura pas assez de personnes pour la remplir. Qui viendra ici ? Qui marchera jusqu'ici ? Vous feriez mieux de changer d'idée! " Je ne l'ai pas écouté parce que je sais qu'il n'y a pas de vent contraire pour celui qui sait où il va. Quand vous avez la conviction de ce que vous faites, personne, absolument personne ne peut vous retenir.

Le pasteur Valentin prit donc le risque. Il mit sa famille en danger. Il vida son compte en banque, avec l'accord de sa femme, pour acheter un terrain. Il reçut des dons de la diaspora ; il reçut l'aide de professionnels, d'un architecte notamment. Il n'attendit pas après ses fidèles pour vivre. C'est un entrepreneur, c'est-à-dire un homme d'action ; un homme engagé ; un homme de conviction. Quand je le rencontrai, il dirigeait toujours son école de langues, présidait la session haïtienne de la Fondation pour la paix et avait un projet très clair pour son église. J'en vis les fondations. Pour le reste, il m'expliqua : là le terrain de basket, là l'école, pour laquelle des professeurs étaient actuellement en formation parce qu'il voulait qu'ils viennent de Ganthier.

Nous avons des jeunes à l'église qui se portent volontaires pour enseigner. Ils travaillent par équipe parce qu'ils comprennent le projet et ils sont prêts à mettre à la disposition de l'église tout ce qu'ils ont comme talent et comme capacité.

Avec l'aide de la Fondation pour la paix, le pasteur Valentin réalisa également des travaux pour améliorer la vie dans la commune et restaurer ce que le séisme avait détruit : canalisation des jardins, réparation de toits, et en attendant que son école soit prête à accueillir les enfants, il participa au financement de la 
scolarisation de dix-sept élèves, grâce aux aides qu'il avait su mobiliser, y compris auprès de l'État. Enfin, il comptait construire une école professionnelle pour permettre aux jeunes d'apprendre un métier et rendre simplement possible leur accès au travail. Il voulait ainsi les aider à devenir des acteurs économiques. Cela nécessitait d'augmenter leur capital humain, ce qui passait par le renforcement de leurs "facteurs physiques [et] psychologiques" ainsi que de leur " aptitude à travailler, leur compétence, leur pouvoir-faire quelque chose " pour que chacun soit en mesure de gagner un salaire (M. Foucault, 2004 : 230). Ce faisant, il introduisait le fidèle dans la logique néolibérale dans une perspective semblable à celle des Églises néo-pentecôtistes éthiopiennes décrites par E. Fantini, qui refusent également la théologie de la prospérité sans pour autant tourner le dos au succès économique.

Le pasteur Valentin : «Une école professionnelle, c'est une école où l'on apprend les métiers. Par exemple, l'électricité, la plomberie, la maçonnerie, l'informatique, les langues. Il n'y en a aucune dans la commune. Tout est concentré à Port-au-Prince [...] Parce qu'il y a une chose que je veux enseigner aux jeunes. Je veux les motiver à comprendre ça. C'est pendant que vous êtes jeunes que vous devez travailler. Vous avez toute votre vigueur. Il y a une chose que je veux enseigner à l'église et surtout aux jeunes de ne pas se croiser les bras à attendre que Dieu intervienne. Je n'aime pas cette idée-là d'attendre toujours que les Français viennent faire quelque chose, que les Américains viennent faire quelque chose, que les Anglais, les Dominicains... C'est à nous de faire des choses. Et surtout, il ne faut pas avoir peur! Celui qui échoue et celui qui n'a pas essayé [...] Moi, j’ai appris à entreprendre beaucoup d'activités ».

C'était le dimanche 11 mai 2014. J'étais en voiture et nous suivions le bus de l'église. Le pasteur vint nous saluer, me prévint que cela allait être un peu long car il devait récupérer les membres de l'église éparpillés dans différents quartiers. On était en pleine campagne sur une route chaotique au milieu de champs de cannes à sucre. Le chauffeur écoutait une radio protestante. Sur place, une cinquantaine de personnes étaient présentes. Elles avaient installé des chaises, un micro, dans une sorte de préau dont les murets délimitaient la future paroisse qui donnait directement sur la montagne. C'était très joli. Apaisant. À l'extérieur, les enfants assistaient à une leçon de catéchisme. À nouveau, je fus frappée par les tenues. On se croyait en centre-ville. Quelle élégance! Quel port altier que celui de ces femmes ! Ma présence n'était pas appréciée de tous, mais l'on m'accepta. Durant le sermon, le pasteur Valentin rappela que la grâce était pour tout le monde. Que le rôle des parents était crucial et qu'ils devaient surveiller les relations et l'éducation de leurs enfants. Se comporter en modèles face à eux. Il rappela aux enfants l'importance d'étudier. Et de préciser :

La charité bien ordonnée commence par soi-même. Construisez-vous. Apprenez à vivre ensemble. Il faut participer à faire changer Haïti. Il faut cesser de vendre des terrains à la diaspora. Il faut respecter l'environnement. Il faut cesser de se soumettre. Il faut chercher la liberté sociale, économique. Il ne faut pas dépendre des autres. J'ai un peu de sous. J'achète des poulets et je vis de cette activité. 
Bien plus que le sermon, ce qui se passa avant et après retint l'attention. D'abord, une petite fille de cinq ou six ans prit la parole et demanda que Jésus bénisse l'assemblée. L'assemblée répondit à sa prière avec le même sérieux qu'à un adulte. Puis elle se mit à chanter. Après le sermon, des adolescents de différents âges jouèrent une pièce de théâtre dont le contenu apportait des réponses à des situations auxquelles ils étaient régulièrement confrontés dans leur vie quotidienne (mauvaises rencontres, tentations, découragement, etc.). Bref, la volonté d'investir sur les enfants était évidente. De leur donner goût au travail, à l'apprentissage, à l'entraide. Cette pièce conclut le culte. On empila les chaises en rigolant, deux jeunes filles plièrent les rideaux en se drapant d'abord avec. Puis la musique démarra. Le préau se transforma en piste de danse. Tout le monde s'amusa et se trémoussa, avec grâce, avec rythme, avec un plaisir non dissimulé. Des plus jeunes aux plus âgés, tous avaient le sourire aux lèvres. C'était une ambiance festive et conviviale, complice, intergénérationnelle qui résonnait dans la campagne, au milieu de nulle part, du moins pour l'instant, car dans les yeux du pasteur Valentin, l'école, le terrain de basket, l'église étaient déjà là. Les fidèles remontèrent dans le bus, qui s'éloigna doucement, se trémoussant lui aussi sur la route chaotique. Aucun doute : tous partageaient le même espoir.

Fonder une nouvelle Église constitue le meilleur moyen de faire se multiplier la communauté de fidèles [...] Les nouvelles Églises sont plus flexibles et s'adaptent plus facilement aux besoins du temps présent. Elles permettent également l'émergence de nouveaux leaders. Et parce que les nouvelles Églises répondent au plus près aux besoins de leurs membres, tels qu'ils s'expriment individuellement, ceux-ci peuvent acquérir plus facilement une identité communautaire, qui découle de leur intériorisation de la "vision" dont le pasteur-leader s'affirme être porteur (J. Garcia-Ruiz, P. Michel, 2012: 98).

La vision du pasteur-leader Valentin avait pour dernier aspect une très forte dimension politique qui ambitionnait le dépassement des clivages religieux. Son action la plus révolutionnaire fut sans doute la place originale qu'il fit aux vaudouisants. Cela ne fut pas sans choquer sa communauté, mais il n'y renonça pas pour autant. Non seulement il paya les frais de scolarité du fils de l'hougan ${ }^{10}$ du village, mais encore, il invita les pratiquants du vaudou à se joindre aux festivités qu'il organisait une fois par mois dans son église avec l'ensemble des villageois qui le désiraient. En cela, il tentait de dépasser les mépris croisés qui, au cours de l'histoire d'Haïti, avaient entaché le partage de l' ' haïtianité » : d'abord celui des élites catholiques face au vaudou ; ensuite celui des élites intellectuelles nombreuses à considérer aujourd'hui le vaudou comme le " ciment réel de la société haïtienne » (Hurbon, 2004 : 260 et 262) face aux Églises évangéliques et pentecôtistes; enfin la diabolisation du vaudou par les protestants, conduisant à un refus des vaudouisants de considérer ces derniers comme des Haïtiens et cela malgré leur croissance continue depuis le début des années 1980

10. Chef spirituel vaudou. 
(A. Corten, 2014). En réponse à ces différents niveaux d'essentialisation de l'appartenance identitaire, le pasteur Valentin proposait de remettre la société en mouvement : il confirmait la résistance face à l'Occident en refusant tout autant la centralité de l'argent et la victimisation dans laquelle les ONG maintenaient les Haïtiens. Il trouvait ainsi un point d'entente possible avec les pratiquants du vaudou. Il tentait par ailleurs de faire bouger la société par le bas et militait pour un projet politique alternatif dans lequel l'État tout comme les ONG jouaient un rôle secondaire. Bref s'il était proche du néo-pentecôtisme, il s'en distinguait en mettant à distance la réussite matérielle et en acceptant la pluralité des croyances. Le pasteur Valentin était un entrepreneur doublé d'un homme politique.

J. P. Warnier s'attache à démontrer que la valeur est l'aboutissement d'un processus de construction. Elle peut être révisée à la hausse comme à la baisse en fonction de l'environnement dans lequel le bien ou la personne est évalué (J. P. Warnier, 2009). Le changement de l'appréciation de sa valeur par le fidèle est le premier travail tenté par les distributeurs de l'entreprise AMWAY comme par les pasteurs des deux dernières églises visitées. À cet égard, la sociologie de la pauvreté de Simmel aide à éclairer les enjeux de cette transformation. Pour lui aussi, la pauvreté est toujours relative. C'est la société qui la construit, lui donne ses contours et d'une certaine façon y enferme les pauvres. Dans le cas d'Haït, c'est plus largement le regard que les puissances mondiales posent sur ce pays qui le réduit à son état de pauvreté. Les pauvres haïtiens sont ceux qui ne peuvent survivre sans l'aide de la collectivité internationale et qui, par conséquent, sont perçus à la fois comme dépendants d'elle et non désirés par elle. Le jugement des ONG et de la MINUSTAH sur le peuple haïtien leur donne une telle ascendance sur lui qu'elles se permettraient parfois l'inacceptable (les habitants dénoncent des viols, des vols, etc.). La pauvreté légitime leur fonction et leur sentiment d'utilité maintient les Haïtiens dans une identité figée, stigmatisante, d'où les sort la démarche responsabilisante des pasteurs. En œuvrant à modifier la structure matérielle, culturelle, sociale de l'environnement immédiat de l'église pour créer les conditions favorables au développement local, ils permettent à chacun de se trouver une fonction au sein de l'église, pour son village ou son quartier, et finalement dans la société. Leur visée est de faire du fidèle un travailleur, c'est-à-dire un sujet économique actif. L'église devient le lieu où le fidèle est encouragé à trouver " une aptitude à travailler, une compétence, un pouvoirfaire quelque chose " (M. Foucault, 2004 : 230); les moyens de devenir un " entrepreneur de lui-même ", sortant dès lors de la logique de l'assistanat à l'origine de «l'exclusion des pauvres » (G. Simmel, $2008: 51$ ).

La communauté de Ganthier est la plus intéressante à cet égard. Des villageois décident d'engager un pasteur qui réponde à leurs besoins pour mettre en action leurs désirs. Il est à la fois employé et chef de l'entreprise communautaire. S'il 
ne va pas dans leur sens, ils s'en iront. Il n'y a pas de patronat; il n'y a pas d'argent non plus. Non pas que l'argent ne soit pas souhaité, mais que les fidèles n'en disposent pas ou si peu qu'il cesse d'être une valeur d'échange. L'argent vient d'ailleurs. Il sert les besoins de la collectivité, mais c'est par son énergie que l'individu est appelé à sortir de la misère. Le pasteur oblige le fidèle à se demander ce qu'il possède en lui-même qu'il pourrait mettre au service de la communauté et de sa propre transformation c'est-à-dire, au service d'une augmentation de sa propre valeur. La mobilisation des fidèles à laquelle il semble parvenir est « une affaire de colinéarité » (F. Lordon, 2010 : 54). Il s'agit d'aligner le désir des fidèles sur une volonté politique au centre de laquelle se trouvent l'éducation des jeunes, la formation des adultes et le respect de la pluralité religieuse. Tout est fait pour développer "le désir intrinsèque de l'activité pour elle-même » et la possibilité de «la réalisation de soi dans et par le travail " (Lordon: 76). Finalement, la croissance et la réussite de l'entreprise paroissiale dépendent de la montée en valeur de ses fidèles. Si, comme l'affirme Spinoza, "pouvoir et faire sont une seule et même chose » (F. Lordon : 183) alors il s'agit assurément pour le pasteur de renforcer la croyance des paroissiens en leur pouvoir.

Le projet du pasteur-entrepreneur-homme politique Valentin est de participer à la création d'une communauté partageant un "destin réalisateur commun ». Dans ce cas, selon F. Lordon, " on peut donner à l'entreprise générale le nom de "récommune", res communa décalquée de la res publica, chose simplement commune puisqu'elle est plus étroite en nombre et en finalités que la chose publique, mais enclave de vie partagée susceptible comme telle d'être organisée selon le même principe que la république idéale : la démocratie radicale " (169). Les paroisses néo-pentecôtistes visitées remettent en cause la présence et les actions des ONG, parce qu'il leur semble indispensable pour la reconstruction de l'individu, de sa famille, du village et finalement du pays, que les fidèles partagent " l'entière maîtrise des conditions de la poursuite collective de l'objet, et [affirment] le droit irréfragable d'être pleinement associés à ce qui les concerne [...] Le simplissime principe récommuniste est donc que ce qui affecte tous doit être l'objet de tous [...], c'est-à-dire constitutionnellement et égalitairement débattu par tous » (170). On veut croire avec F. Lordon que l'éruption d'indignation provoquée par la façon dont les ONG et la MINUSTAH ont pris possession de la nation haïtienne " rencontrera une cristallisation affective sur laquelle, si petite soit-elle à l'origine, elle produira des effets de précipitation catalytique » (179). En même temps, et pour aller dans l'autre direction, il apparaît que l'entreprise économico-politico-religieuse de Valentin n'est pas sans rapport avec la définition même de l'entreprise que Foucault repère dans le néolibéralisme. Il ne s'agit pas en effet de créer des multinationales mais au contraire de faire en sorte que chaque " unité de base » (l'individu lui-même, sa famille, etc.) se développe sous la forme d'une entreprise de telle sorte que celle-ci devienne « la puissance informant la société » (M. Foucault, 2004 : 154). Or cela n'est possible que si les activités économiques ainsi mises en place sont réglées. "Ces règles, précise 
Foucault dans sa leçon du 21 février 1979, ça peut être un habitus social, une prescription religieuse, ça peut être une éthique, ça peut être un règlement corporatif, ça peut être une loi. "En l'absence de lois solidement posées par l'État, c'est l'habitus social que les pasteurs néo-pentecôtistes présentés dans cet article essaient de transformer, à travers la prescription religieuse. Et en cela, ils jouent le jeu du néolibéralisme.

Nathalie LUCA

Centre d'études en sciences sociales du religieux (CéSor)

UMR 8216, CNRS-EHESS

nathalie.luca@ehess.fr

\section{Bibliographie}

ANDERSON Benedict, 1996, L'imaginaire national. Réflexions sur l'origine et l'essor du nationalisme, Paris, La Découverte.

Aubin-Boltanski Emma, Lamine Anne-Sophie, Luca Nathalie, 2014, Croire en actes: distance, intensité, excès, Paris, L'Harmattan, "Religions en questions ».

Certeau de Michel, 1990, L'invention du quotidien. 1. Arts de faire, Paris, Gallimard, «Folio essais ».

Clorméus Lewis Ampidu, 2012, Entre l'État, les élites et les religions en Haïti. Redécouvrir la campagne antisuperstitieuse de 1939-1942, doctorat de sociologie, Paris, École des hautes études en sciences sociales.

-, 2014, "La situation religieuse en Haïti après le séisme du 12 janvier 2010. Entretien avec Lewis Ampidu Clorméus ", Histoire, monde et cultures religieuses, n 29, p. 133-136.

CORTEn André, 2001, Misère, religion et politique en Haïti. Diabolisation et mal politique, Paris, Karthala.

-, 2014, "Pentecôtisme, baptisme et système politique en Haïti ", Histoire, monde et cultures religieuses, $\mathrm{n}^{\circ} 29$, p. 119-132.

Foucault Michel, 2004, Naissance de la biopolitique. Cours au Collège de France 19781979, Paris, Hautes études, École des hautes études en sciences sociales, Gallimard, Le Seuil.

Garcia-Ruiz Jesus, Michel Patrick, 2012, Et Dieu sous-traita le salut au marché. De l'action des mouvements évangéliques en Amérique Latine, Paris, Armand Colin, «Recherches".

GIRARD René, 1987, Violent Origins. Walter Burkert, René Girard and Jonathan Z. Smith on Ritual Killing and Cultural Formation, Stanford, Stanford University Press.

Hurbon Laënnec, 2004, Religions et lien social. L'Église et l'État moderne en Haïti, Paris, Le Cerf.

Lordon Frédéric, 2010, Capitalisme, désir et servitude. Marx et Spinoza, Paris, La Fabrique.

Luca Nathalie, 2008, Individus et pouvoirs face aux sectes, Paris, Armand Colin, "Sociétales ».

-, 2011, "Multi-Level Marketing: At the Crossroads of Economy and Religion ", Research in Economic Anthropology, vol. 31, p. 219-241. 
-, 2012, Y croire et en rêver. Réussir dans le marketing relationnel de multiniveaux, Paris, L'Harmattan, "Religions en questions ».

McAlister Elisabeth, 2013, «Humanitarian Adhocracy, Transnational New Apostolic Missions, and Evangelical Anti-Dependency in a Haitian Refugee Camp ", Nova Religio: The Journal of Alternative and Emergent Religions, vol. 16, Issue 4, p. 11-34.

Richman Karen E., 2005, Migration and Vaudou, Florida, University Press of Florida. Simmel Georg, 2008, Les pauvres, Paris, Presses universitaires de France, "Quadrige ». WARnier Jean-Pierre, 2009, "Les politiques de la valeur ", Sociétés politiques comparées, $\mathrm{n}^{\circ} 11$, http://www.fasapo.org, p. 1-67.

Woods Paolo, Robert Arnaud, 2014, Leta, Port-au-Prince, Fokal Éditions.

\section{Pentecôtismes et esprit d'entreprise en Haïti}

Le séisme du 10 janvier 2010 à Port-au-Prince a modifié le paysage religieux haïtien qui a vu nettement progresser les églises néopentecôtistes. Celles-ci ne sont certes pas animées par une théologie unifiée et au-delà de quelques pratiques communes, les attentes des fidèles et les prêches des pasteurs sont très variés. On peut observer le développement d'une théologie sociale dont la portée, à la fois politique et économique, tente de combler, au petit niveau de la communauté religieuse, l'absence de l'État. Cette théologie participe de ce souffle nouveau qui pousse à la création d'une "Haïti nouvelle» et cherche à remettre les Haïtiens sur la voie du travail, de l'enrichissement et de l'indépendance, en particulier vis-à-vis des ONG.

Mots-clés : Haïti, églises néopentecôtistes, théologie sociale, conatus et désir, Frédéric Lordon.

\section{Pentecostalisms and entrepreneurial spirit in Haiti}

The earthquake of January 10th, 2010 in Port-au-Prince modified the Haitian religious landscape with a clear progress of the neoPentecostal churches. These are not led by a unified theology and, beyond some common practices, the expectations of the believers and the sermons of the ministers are highly varied. We can observe the development of a social theology which impact, at the same time political and economic, tries to fill the absence of the State at the religious community's level. This theology belongs with this new breath which pushes to the creation of a "new Haiti" and tries to put the Haitians back on the way to work, enrichment and independence, in particular regarding non-governmental organizations.

Keywords: Haiti, neoPentecostal churches, social theology, conatus and desire, Frédéric Lordon.

\section{Pentecostalismos y espíritu de empresa en Haití}

El sismo del 10 de enero de 2010 en Port-au-Prince modificó el paisaje religioso haitiano, que vió progresar claramente las iglesias neo-pentecostales. Éstas no comparten por cierto una teología unificada, y más allá de algunas prácticas comunes, las expectativas de los fieles y las prédicas de los pastores son muy variadas. Se 
puede observar el desarrollo de una teología social cuyo alcance, a la vez político y económico, trata de subsanar, al nivel reducido de la comunidad religiosa, la ausencia del Estado. Esta teología participa del aire nuevo que busca la creación de un "nuevo Haití" y devolver a los haitianos al camino del trabajo, del enriquecimiento y de la independencia, en particular frente a las ONG.

Palabras clave: Haití, iglesias neo-pentecostales, teología social, conato y deseo, Federico Lordon. 
